\title{
Los Objetivos de Desarrollo Sostenible como marco para la acción y la intervención social y ambiental
}

\section{The Sustainable Development Goals as frame for the action and social and environmental intervention}

Dr. Daniel Rodrigo-Cano es investigador de la Universidad de Sevilla (España) (daniel.rodrigo@alu.uhu.es) (https://orcid.
org/0000-0003-2753-5470)

Dra. Maria Josep Picó es docente e investigadora de la Universitat Jaume I (España) (mpico@uji.es) (https://orcid.org/00000002-7086-2269)

Dra. Glenda Dimuro es investigadora de la Universidad de Sevilla (Brasil) (glenda.dimuro@gmail.com) (https://orcid.org/00000001-8655-7069)

\begin{abstract}
Resumen
El cambio climático se ha erigido como uno de los mayores riesgos para la vida de todas las especies en la tierra, especialmente para el ser humano quien, a su vez, es su principal causante. La presente investigación busca analizar el papel de los Objetivos de Desarrollo Sostenible (ODS) de las Naciones Unidas para la acción y la intervención social y ambiental y el papel que la educomunicación ambiental tiene ante el reto del cambio climático, como uno de los fenómenos que, por su urgencia, está en la agenda ambiental, social, económica y política. Para ello se ha realizado una revisión bibliográfica actual de las principales investigaciones en el área. A lo largo de este trabajo se ha observado que el cambio climático y los ODS, definidos por entidades supranacionales, no están presentes en la educación ambiental y que el ámbito de la comunicación ambiental se centra, especialmente, en las catástrofes y situaciones extremas. En el actual entorno digital, donde la inmediatez de las redes sociales ha favorecido la difusión de informaciones falsas, es necesario la recuperación de enfoques críticos e ideológicos de la educación para los medios, para el desarrollo de la educación mediática y de la competencia digital con el fin de promover una ciudadanía con capacidad para construir nuevas narrativas a través de los medios digitales, para con ello facilitar nuevas formas de «ecociudadanías» capaces de provocar un cambio necesario y transformador en el sistema.
\end{abstract}

\begin{abstract}
Climate change has emerged as one of the most important risks for citizenships and especially for the human being, who is known as the main cause of this change. The aim of this research is to analyze the role of sustainable development objectives for the action and social and environmental intervention and the role that environmental educommunication has with the challenge of climate change, as one of the phenomena that, by its urgency, is on the environmental, social, economic and political agenda of organizations. Thus, a bibliographical review of the main investigations in the area was carried out in which it was observed that climate change and the objectives of sustainable development, defined by supranational entities, are not present in environmental education, and only special interest is paid to the catastrophes and extreme situations in environmental communication. In times of social networking and false information, the recovery of critical and ideological approaches to media education is necessary for the development of media education and digital competence to be able to create new narratives through digital media for new forms of ecocitizenship capable of causing a necessary change and transforming the system.
\end{abstract}

\section{Palabras clave I keywords}

Cambio climático, objetivos de desarrollo sostenible, redes sociales, educomunicación, ecociudadanía.

Climate change, sustainable development goals, digital ecosystem, social networks, educommunication, ecocitizenship.

Cómo citar: Rodrigo-Cano, D. Picó, M.J. y Dimuro, G. (2019). Los Objetivos de Desarrollo Sostenible como marco para la acción y la intervención social y ambiental. Retos Revista de Ciencias de la Administración y Economía, 9(17), 25-36. https://doi.org/10.17163/ret.n17.2019.02 


\section{Los Objetivos de Desarrollo Sostenible (ODS)}

Las Naciones Unidas aprobaron los Objetivos de Desarrollo Sostenible (ODS) en 2015, tras la década de la Educación para el Desarrollo Sostenible (2004-2015) impulsada por la Unesco y los Objetivos del Desarrollo de Milenio (2005-2015). Estos son los 17 Objetivos de Desarrollo Sostenible: 1) Pobreza, 2) Hambre cero; 3) Salud bienestar; 4) Educación; 5) Igualdad de género; 6) Agua; 7) Energía asequible y no contaminante; 8) Trabajo decente; 9) Industria, innovación e infraestructura; 10) Reducción de las desigualdades; 11) Ciudades y Comunidades Sostenibles; 12) Consumo responsable; 13) Acción por el clima (cambio climático); 14) Vida marina; 15) Ecosistemas terrestres; 16) Paz, justicia e instituciones sólidas; 17) Alianzas (Programa de las Naciones Unidas para el Desarrollo, 2016). Estos Objetivos de Desarrollo Sostenible permiten un marco referencial para la acción y la intervención social y ambiental, a pesar de una evidente falta de integración de variables sociales (Allen, Metternicht, \& Wiedmann, 2016).

Figura 1. Objetivos de Desarrollo Sostenible

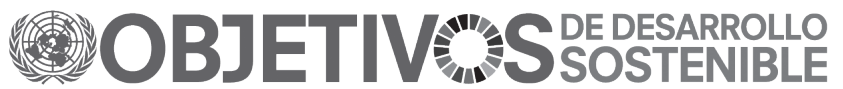


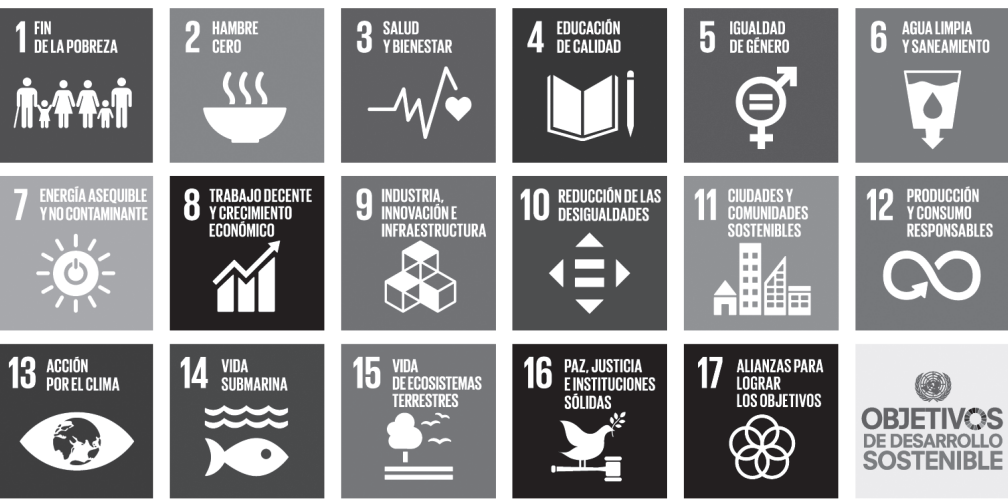

Fuente: Organización de Naciones Unidas (2017)

Desde los ODS se plantean ciertas metas para el 2030, que pasan por poner fin a la pobreza y mejorar los accesos a la educación, al empleo y la información, mejorar la salud y la vivienda y la reducción de las desigualdades al avanzar hacia el consumo y la producción de una alimentación sostenible. De la misma forma, considera necesario asegurar la seguridad hídrica, una energía limpia universal, ecosistemas saludables y productivos y gobernanza para sociedad sostenibles (Griggs, 2013).

En definitiva, el «desarrollo sostenible» consiste en una práctica que incluye el impulso de movimientos sociales, la organización de las instituciones, la elaboración de la ciencia y la tecnología y la negociación de compromisos entre quienes se preocupan por el medio ambiente, la economía y los aspectos sociales (Robert, Parris, \& Leiserowitz, 2005). 
En este sentido, resulta imperativo que la «educomunicación ambiental» transite hacia el desarrollo sostenible para alcanzar los ODS. Para ello, la educación ambiental y la comunicación ambiental deben estar alineadas con el fin de señalar la relevancia de la calidad de las campañas de sensibilización o la educación ambiental en la comunicación desarrolladas tanto por asociaciones como por instituciones. Se ha de evitar caer en tópicos e improvisaciones y fundamentar los mensajes y el diseño metodológico en la información científica (Picó, 2017a).

En la presente revisión se ha llevado a cabo una selección de trabajos publicados en las bases de datos Web of Science ${ }^{\circledR}$ y Scopus ${ }^{\circledR}$ de los últimos años, partiendo de los criterios de búsqueda sobre "Objetivos de Desarrollo Sostenible» [Sustainable Development Goals] y teniendo en cuenta que se tratan de las principales y más prestigiosas bases de datos científicas a escala internacional, lo que asegura la calidad de los trabajos indexados.

Para llevar a cabo el análisis de contenido propuesto en este estudio, y teniendo en cuenta las fases lógicas en este tipo de aproximaciones, se establece un procedimiento que se ajuste a los objetivos planteados. En la primera fase de búsqueda y revisión de literatura se seleccionó el término «Sustainable Development Goals» y «Objetivos de Desarrollo Sostenible» (con algoritmos booleanos entre comillas y con asteriscos) en el periodo comprendido 2011-2018, con la finalidad de acotar aquellos trabajos emergentes que hiciesen referencia específicamente a los Objetivos de Desarrollo Sostenible. Posteriormente, se refinaron los datos obtenidos en función del tipo de documento, seleccionando únicamente los artículos y excluyendo capítulos de libros, las revisiones o reseñas, las actas de congresos y los artículos en prensa, con el fin de reducir el número de documentos (tabla 1).

\section{Tabla 1. Documentos emergentes por año por criterios

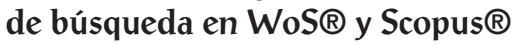

\begin{tabular}{|c|c|c|}
\hline Año & SDG $^{*}$ & ODS $*^{*}$ \\
\hline 2011 & 31 & \\
\hline 2012 & 27 & \\
\hline 2013 & 44 & \\
\hline 2014 & 47 & 2 \\
\hline 2015 & 88 & 3 \\
\hline 2016 & 154 & 8 \\
\hline 2017 & 258 & 13 \\
\hline 2018 & 359 & \\
\hline TOTAL & 1008 & \\
\hline
\end{tabular}

*SDG $=$ Sustainable Development Goals; $* *$ ODS = Objetivos de Desarrollo Sostenible 


\section{Educomunicación ambiental}

Son necesarias herramientas que permitan una «alfabetización ambiental», así como una educomunicación capaz de desarrollar la capacidad crítica y el empoderamiento de la ecociudadanía, entendida ésta como aquella que sirve para "reforzar la libertad, la autonomía crítica y la participación de los ciudadanos en cuestiones políticas, sociales, económicas, ecológicas e interculturales a partir del buen uso de los medios y la tecnología comunicativa" (Gozálvez y Contreras-Pulido, 2014: 130). Hace falta una colaboración en la búsqueda de respuestas para la mitigación y la adaptación ante el cambio climático, por lo que resulta necesario la implicación en la participación de diferentes actores en la transformación (Sarmiento, 2013).

La educomunicación ambiental debe ser entendida como una capacitación para la acción y caracterizada por su intencionalidad, por la implicación de la sociedad, explicando los motivos y las razones así como los mecanismos y las causas desde la ciencia. Para ello es necesaria la búsqueda de soluciones, la participación democrática y la visión de un futuro utópico en los que la salud humana, los conflictos sociales o la equidad deben ser muy destacados y para ello se ha poner énfasis en la acción (Rodrigo-Cano \& Machuca-De-La-Rosa, 2018).

Más allá de la educomunicación como reflexión dialógica y emancipatoria que propone Paulo Freire para la construcción colaborativa del conocimiento, encaminada al empoderamiento y al ejercicio de la ciudadanía, y por extensión de la ecociudadanía, es necesario un proceso que favorezca el desarrollo de competencias en la percepción e interpretación de los mensajes que hoy posibilitan las tecnologías digitales emergentes (smartphones, tablets y phablets) (Nagamini \& Aguaded, 2018), y en el que la educomunicación se caracteriza por factor relacional dentro del ecosistema digital a través de la comunicación digital, las interacciones, las habilidades psicosociales de la ciudadanía en los entornos sociales, de ocio y lúdicos y de conciencia crítica (Marta-Lazo \& Gabelas, 2016) que, en muchas ocasiones, se desarrollan a través de herramientas y medios digitales (Villalonga \& Marta-Lazo, 2015).

Ante la evidencia de la existencia de un cambio climático en curso y en el que la única variable que puede explicarlo es la interferencia humana en la atmósfera (IPCC, 2013), los expertos reclaman "limitar el calentamiento a $1,5{ }^{\circ} \mathrm{C}$ requiere un cambio transformador sistémico” (IPCC, 2018). En esta línea se hace urgente que desde todas las disciplinas se busquen soluciones viables, realistas y que supongan un compromiso político con las personas ciudadanas.

Los impactos que los expertos ya identifican pasan por la disminución de los glaciares alterando el ciclo hidrológico, aumento de temperaturas alterando ecosistemas terrestres y acuáticos (extinción), aumento de las sequías y mayor riesgo de incendio, aumento del nivel del mar, estrés por calor, precipitaciones extremas, inundaciones, sequía, aumento de la aridez y escasez de agua, evolución negativa de los rendimientos de determinados cultivos, entre otros muchos efectos que coligen un llamado de atención.

Pero los mayores efectos del calentamiento global se van a producir sobre la especie humana. Sus consecuencias van a modificar los patrones de las enfermedades transmitidas por mosquitos y garrapatas o aumentar las enfermedades transmitidas 
por el agua, debido a condiciones más cálidas y cambios en las precipitaciones y la escorrentía. Además, a partir de impactos sobre los sistemas sociales, también incidirá en aspectos como la seguridad alimentaria, la capacidad laboral, la salud mental, el desplazamiento de la población y otros efectos sobre los sistemas de atención de la salud (Ministerio de Agricultura, Alimentación y Medio Ambiente [España], 2014). En resumen, si la humanidad continua el actual ritmo de emisiones de efecto invernadero, las consecuencias del cambio climático pueden ser catastróficas para la humanidad y, especialmente, para las sociedades y los grupos humanos más vulnerables (Vervoort \& Gupta, 2018).

Para hacer frente al cambio climático, tanto las organizaciones gubernamentales como las organizaciones sociales y ciudadanas plantean estrategias adaptativas de reducción de riesgos ante el cambio climático a través de un mejor acceso a la educación y la información, la nutrición, los servicios sanitarios, la energía, la vivienda segura y las estructuras de asentamiento y de apoyo social, así como un mejor acceso a los recursos locales y control de éstos. Asimismo resulta fundamental el acceso a la tecnología y foros de toma de decisiones, lo que conllevaría a una mayor capacidad de consensos y acuerdos sociales. De la misma forma es necesario atender a opciones tecnológicas, ecosistémicas, económicas y de servicios, a través de leyes y regulaciones con políticas y programas nacionales y gubernamentales, por supuesto desde una menor desigualdad de género a otras formas de marginación (Ministerio de Agricultura, Alimentación y Medio Ambiente [España], 2014).

En este proceso de transformación encontramos a los medios de comunicación que, como indica Meira (2017, p. 98), aún son:

[la] fuente principal de información sobre el cambio climático que tienen los ciudadanos, por lo que recae en ellos una considerable responsabilidad social, aunque hay que anotar también en su descargo sus dificultades estructurales, en un proceso irreversible de cambio hacia los formatos digitales y por la información que los usuarios generan y distribuyen en las redes sociales, además de las limitaciones fruto de los vaivenes políticos y mediáticos de cada momento.

Sin embargo, los expertos advierten que los medios de comunicación ponen el foco en la importancia de las consecuencias del cambio climático en:

[...] el hábitat, la fauna, la flora y los seres vivos, donde las noticias se circunscriben en el cambio de los ciclos naturales de los animales y la vegetación, la subida de las temperaturas y sus consecuencias para los ciclos naturales y la salud, las olas de acciones climatológicas adversas y la falta de lluvias y sus efectos, pero siempre desde un punto de vista pesimista, dado que sólo se relatan las negativas y devastadoras consecuencias que ello produce (Zaragoza, 2018, p.42).

Estas fórmulas de comunicación sobre el cambio climático tiene efectos directos en la acción, reacción y formación de la realidad social, pues la forma en que estos temas se tratan en los medios de comunicación puede tener consecuencias de alcance en la investigación científica sobre el clima, así como en las percepciones, la comprensión del problema y la implicación potencial de responsables políticos y ciudadanos (Boykoff \& Smith, 2010). Dada la situación en la que se encuentran los 
profesionales de la Comunicación, cuyo índice de desempleo en España registrado entre los periodistas en 2016 es un 74\% superior al de 2008 (Asociación de la Prensa de Madrid, 2016), la post-verdad se posiciona con fuerza desde las redes sociales hacia la pseudociencia y oculta los contenidos científicos, como la negación del cambio climático, posicionando la tecnología en el eje naturaleza-sociedad como tabla de salvación.

Esta fragilidad en la información ambiental en general, y sobre el cambio climático en particular, se visibiliza en las relaciones de poder político-económico con los medios de comunicación (Reig \& Labio, 2017; Cuevas \& Mora, 2016). Por otro lado, "las buenas noticias no son noticias" (Picó, 2017b), mientras los medios de comunicación se centran en los riesgos y las catástrofes ambientales (Jiménez \& Martín-Sosa, 2018; Fernández-Reyes, 2018).

Con todo, hay una proliferación de noticias falseadas (fake news), «ecopostureos» (greenwashing) y comunicación infoxicada por algoritmos y bigdata, apoyados por medios de comunicación de masas y sus anunciantes, que pretenden vender productos «verdes» como medio de lavado de cara, cuando su finalidad real no es comprometerse con el entorno (ni ambiental, ni social ni cultural) (Delmas \& Burbano, 2011), se trata de hacer percibir a los consumidores que los productos son ecológicamente amables (Megías-Delgado, Baldallo-González, \& Maraver-López, 2018).

\section{Nuevas narrativas para la educomunicación ambiental}

En este contexto aparecen nuevas narrativas para promover un cambio social hacia un nuevo paradigma (Teso, 2016). En palabras de Naomi Klein (2015): el cambio climático es la narrativa más poderosa contra el sistema económico y político actual. Aunque el cambio climático ha sido desplazado del debate social precisamente por consideraciones económicas y políticas (citado por León \& Bourk, 2018).

Las nuevas formas de comunicación han irrumpido con el uso de Internet y en estos momentos se caracterizan por la descentralización en la emisión de mensajes, la relación entre iguales que mantienen los interlocutores y, a la vez, ser sujetos de la comunicación que emiten y recepcionan (emirecs) (Aparici \& García-Marín, 2018). Esto ocurre en un ecosistema digital, caracterizado por la relación entre la actividad comunicacional, los contenidos y los sujetos de la comunicación (Alonso, 2005) que establecen lógicas, procesos y dinámicas comunicativas que permiten un flujo comunicativo a través de los medios digitales a través de un relato transmediático (Carrera et al., 2013).

Este ecosistema digital y, de forma más genérica, la divulgación científica, requieren la creación de nuevos contenidos relativos al cambio climático para la emisión de forma periódica y el desarrollo de nuevos formatos de contenido divulgativo, apoyados por contenidos de ficción, con la intención de incidir en cambios de actitudes, estilos de vida y comportamientos (Teso Alonso et al., 2018).

Además, han aparecido narrativas transmedia a través de las redes sociales (Ojeda-Barceló, Gutiérrez-Pérez, \& Perales-Palacios, 2011), un buen ejemplo es el movimiento educomunicador que surgió en el año 2014 con la idea de lanzar la Educación Ambiental. En las redes sociales y consiste fundamentalmente en un 
encuentro mensual a través de Twitter, siguiendo el hashtag \#EA26, donde se debaten temáticas de relevancia para la EA. Es un punto de encuentro abierto de intercambio, de colaboración y enriquecimiento (empoderamiento) referente del sector en la red (Gutiérrez Bastida, 2018; Toboso, De-Casas-Moreno, \& Rodrigo-Cano, 2018; Benayas et al., 2017).

El vídeo digital, con Youtube como modelo, se ha convertido en una herramienta fundamental para la comunicación científica y el cambio climático (León \& Bourk, 2018). Los videojuegos constituyen también una nueva forma de acción social que permite a los jóvenes aprender a proteger el planeta, aprendiendo tópicos y motivando a la acción frente al cambio climático (Ouariachi, Olvera, \& Gutiérrez, 2017). Ante el silencio mediático sobre el cambio climático (Mancinas, 2012) es necesario un periodismo ambiental y sostenible capaz de dotar de una cobertura periodística veraz al respecto de esta temática (Fernández-Reyes, 2004; Fernández-Reyes, PiñuelRaigada, \& Vicente-Mariño, 2015) en donde los medios de comunicación desempeñen un destacado papel en la interpretación de acontecimientos, ejerciendo a la vez como testigos y como actores. A partir de esta función de construcción de la realidad social, seguimos la «teoría de la amplificación del riesgo», la cual vincula la experiencia social del riesgo al volumen de cobertura en la prensa (Fernández-Reyes, 2018).

\section{Ecociudadanía}

El panorama actual nos sitúa ante sectores de la educación y la comunicación que imponen discursos hegemónicos e instrumentales. Un sistema educativo tradicional, estructurado sobre ideologías mercantilistas y neoliberales e instituciones educativas politizadas (Badillo \& Martínez-Roa, 2014). Además, el tratamiento curricular del cambio climático en los planes formativos está siendo invisibilizado y contaminado con prejuicios y estereotipos (Serantes, 2017).

Desde el sector de la Educación Ambiental se empieza a reclamar un currículo de emergencia climática (Meira-Cartea et al., 2018; Resclima, 2018), el desarrollo de habilidades digitales y tecnológicas capaces de establecer vínculos entre la sociedad y la tecnología que permitan participar en procesos participativos en los que las personas incorporemos criterios de sostenibilidad, feminismos y justicia global en las decisiones que se tomen (Machuca \& Rodrigo-Cano, 2018), con el fin de conseguir una cultura del cuidado del clima (Heras, 2016) que, irremediablemente, pase por establecer líneas de trabajo con población excluida como mujeres, niñas (Limón \& Solís, 2014).

La educación ambiental reclama una «ecociudadanía» (Sauvé \& Asselin, 2017) que se caracterice por: un proyecto educativo que invite a clarificar la visiónd el mundo, a tener una mirada crítica hacia realidades socioecológicas, a redefinir la economía y a desarrollar un poder-hacer para reconstruir los lazos entre sociedad y naturaleza, y que además incluya la justicia social (Murga-Menoyo, 2018) y desde el feminismo (Herrero, 2014).

Un excelente ejemplo de ecociudadanía y educomunicación ambiental es el denominado «Confint», campaña pedagógica que atrae la dimensión de la política ambiental hacia la educación. Es un proceso constructivo que moviliza y compromete a los y las jóvenes y a la comunidad educativa en el cual las personas se reúnen 
en distintos niveles organizativos y deliberan sobre la crisis ambiental entendida de manera amplia (ecológica, social, cultural, económica, política...), asumen responsabilidades, plantean y ejecutan acciones transformadoras hacia sociedades sostenibles y eligen representantes que llevan a las «Confint» de sucesivos niveles como son el regional, nacional o internacional.

La «Confint» es un proceso: 1) pedagógico, que profundiza en conceptos y valores sobre eco-ciudadanía, medio ambiente, democracia y participación; 2) educativo ambiental, que busca el compromiso y la responsabilidad de las chicas y chicos frente a la crisis ambiental que sufre nuestro planeta; 3) interactivo entre jóvenes de diferentes edades y de distintas regiones, países o continentes que aprenden y actúan unidos y que tienen un fin común: cuidar el planeta; que aproxima la dimensión de la política ambiental hacia la educación formal (Gutiérrez Bastida, 2014).

\section{Discusión y conclusiones}

A lo largo de este recorrido de literatura científica respecto a la emergente educomunicación ambiental frente al cambio climático, queda en evidencia la inexorable convergencia entre educación y comunicación, entendiendo esta como el aprendizaje de los recursos que en estos momentos ofrecen los medios de información y comunicación desde las tecnologías. Además se debe recuperar conceptos de la educación ambiental, como la generación de actitudes y aptitudes, para lograr equilibrios entre el ser humano y el entorno, participar en la integración de la propia educomunicación y las soluciones políticas, sociales, económicas, ambientales y éticas con la intención de prevenirlas, minimizarlas o normalizarlas y, ante el cambio climático, la mitigación y adaptación, y partir de la ciencia y la investigación capaces de gestionar problemáticas del entorno y para el ser humano (Meira et al., 2018).

Además, en la actualidad, la educomunicación ambiental no desarrolla muchos de estos los objetivos de desarrollo sostenible, dejando patente la dimensión ambiental sobre la vertiente social y, por supuesto, dejando de lado la dimensión económica. Sin embargo, como se ha visto en esta investigación, los Objetivos de Desarrollo Sostenible constituyen un marco ético y por el bien común (FernándezReyes \& Águlla-Coghlan, 2017).

Sin embargo, es cierto, que la educación ambiental y la comunicación ambiental han estado más ocupadas en una crisis económica que prácticamente desmanteló el sector, castigado con despidos, cierres y pérdidas de bagajes y conocimientos (Benayas et al., 2017; Meira, Barba, \& Castiñeiras, 2017).

El diseño de los mensajes, de las campañas de sensibilización, de la educación ambiental, así como para la comunicación ambiental, se debe fundamentar en la investigación científica basada en los conjuntos de datos, bibliografías y repositorios de investigación, así como del conjunto de especialistas, comunidades de práctica y aprendizaje y los principales acontecimientos científicos (Peña-López \& PadróSolanet, 2017). Son los medios de comunicación los que deben ser canales adecuados para el conocimiento de la situación, dejando de lado el sesgo informativo por el que solo informan de catástrofes y malas noticias (Zaragoza, 2018). 
En todo caso, la educomunicación ambiental debe asumir un perfil fundamentado en la adquisición de conocimientos básicos y del aprendizaje de informaciones pertinentes. Esto permitirá la mejora de fenómenos y problemáticas ambientales para la toma de decisiones adecuadas, la búsqueda de resolución de problemas reales, el desarrollo de las capacidades intelectuales, que posibilitaránla formulación y la operación de soluciones con la intención de promover desarrollos para una visión crítica capaz de poner el énfasis en los procesos de percepción y fomentar el desarrollo de competencias educativas de los sujetos para la educación y la formación de individuos para potenciar cambios de actitudes, valores, prácticas y comportamientos (Rodrigo-Cano \& Machuca-De-La-Rosa, 2018).

Además, es necesario la recuperación de enfoques críticos e ideológicos de la educación para los medios, para el desarrollo de la educación mediática y de la competencia digital (Aparici \& Tyner, 2012). Ante el avance del paradigma digital y el uso de la tecnología móvil, en crecimiento exponencial, se requieren nuevos roles, nuevos formatos y nuevas narrativas que proponen los nuevos medios digitales tanto de la comunicación como de la educación ambiental ante esta realidad comunicativa diversa y caracterizada por los emirecs.

\section{Referencias}

Allen, C., Metternicht, G., \& Wiedmann, T. (2016). National pathways to the Sustainable Development Goals (SDGs): A comparative review of scenario modelling tools. Environmental Science $\mathcal{E}$ Policy, 66, 199-207. http://dx.doi.org/10.1016/j.envsci.2016.09.008

Alonso, J. (2005). Propuesta metodológica para el estudio de las formas de comunicación en internet. En López García, G. (Ed.). El ecosistema digital: Modelos de comunicación, nuevos medios y público en Internet. (pp. 89-107). Valencia: Servei de Publicacions de la Universitat de València.

Aparici, R., \& García-Marín, D. (2018). Prosumers and emirecs: Analysis of two confronted theories. [Prosumidores y emirecs: Análisis de dos teorías enfrentadas]. Comunicar, 55, 71-79. https://doi.org/10.3916/C55-2018-07

Aparici, R. \& Tyner, K. (2012). Educación para los medios, alfabetización mediática y competencia digital. Comunicar, 38, 51- 58. DOI:10.3916/C38-2011-02-05.

Asociación de la Prensa de Madrid (2016). Informe Anual de la Profesión Periodística (2016). Recuperado de https://goo.gl/iwPtRD.

Badillo, M.E., \& Martínez-Roa, O.G. (2014). Educomunicación y medio ambiente: en la búsqueda y construcción de fisuras. Revista de Investigación Agraria y Ambiental, 5(1), 255-270

Benayas, J., Marcén, C., Alba, D., \& Gutiérrez, J.M. (2017). Educación para la sostenibilidad en España. Reflexiones y propuestas. Madrid: Fundación Alternativas y Red Española para el Desarrollo.

Boykoff, M., \& Smith, J. (2010). Media representations of climate change. En Constance Levertracy-Tracy (Ed.), Routledge Handbook of Climate Change and Society (pp. 210-218). Routledge.

Carrera-Alvarez, P., Limón-Serrano, N., Herrero-Curiel, E., \& Sainz de Baranda, C. (2013). Transmedialidad y ecosistema digital. Historia y Comunicación Social,18, 535-545.

Cuevas, R., \& Mora, A. (2016). Latifundio mediático y resistencias sociales en América Latina. San José, Costa Rica: Editorial Universitaria Estatal a Distancia.

Delmas, M. A., \& Burbano, V. (2011). The drivers of Greenwashing. California Management Review, 54(1), 64-87. https://doi.org/10.1525/cmr.2011.54.1.64

Fernández Reyes, R. (2004). Periodismo ambiental y periodismo sostenible. Ámbitos, 12, 311-317. 
Fernández-Reyes, R. (2018). Trump, eventos extremos y cumbres internacionales en la cobertura mediática del cambio climático. En R. Fernández-Reyes \& D. Rodrigo-Cano (Eds). La comunicación de la mitigación y la adaptación al cambio climático (pp. 13-47). Madrid: Ediciones Egregius.

Fernández-Reyes, R., \& Águila-Coghlan, J.C. (217). Un periodismo en transición ante el V informe del IPCC, El acuerdo de París y Los objetivos de desarrollo sostenible. Ámbitos, 37. Recuperado de https://goo.gl/Sj4Zyg

Fernández-Reyes, R., Piñuel-Raigada, J.L., \& Vicente-Mariño, M. (2015). La cobertura periodística del cambio climático y del calentamiento global en El País, El Mundo y La Vanguardia. Revista Latina de Comunicación Social, 70, 122-140. DOI: 10.4185/RLCS-2015-1038

Griggs, D. (2013). Sustainable development goals for peopel and planet. Nature, 495, 305-307.

Gozálvez, V., \& Contreras, P. (2014). Empowering Media Citizenship through Educommunication. [Empoderar a la ciudadanía mediática desde la educomunicación]. Comunicar, 42, 129-136. https://doi.org/10.3916/C42-2014-12

Gutiérrez Bastida, J.M. (2014). La Confint y Esenred: Crisoles de aprendizaje hacia la Eco-ciudadanía. Boletín del CENEAM, número de noviembre. Recuperado de https://goo.gl/SM471e

Gutiérrez Bastida, J.M. (2018). Educatio ambientalis. Invitación a la educación ecosocial en el Antropoceno. Madrid: Editorial Bubok.

Heras, F. (2016). La educación en tiempos de cambio climático: facilitar el aprendizaje para construir una escuela de cuidado del clima. Recuperado de https://goo.gl/3cUjsQ.

Herrero, Y. (2014). Economía ecológica y economía feminista: un diálogo necesario. En Carrasco Bengoa, Cristina (Ed.), Con voz propia. La economía feminista como apuesta teórica y política (pp. 219-237). Madrid: La oveja roja.

IPCC (2014). Climate change 2013: the physical science basis. Recuperado de https://goo.gl/LV1eG4

IPCC (2018). Summary for Policymakers. In: Global warming of $1.5^{\circ} \mathrm{C}$. An IPCC Special Report on the impacts of global warming of $1.5^{\circ} \mathrm{C}$ above pre-industrial levels and related global greenhouse gas emission pathways, in the context of strengthening the global response to the threat of climate change, sustainable development, and efforts to eradicate poverty. Recuperado de https://goo.gl/nJJnH7

Jiménez, I., \& Martín-Sosa, S. (2018). El estudio del cambio climático en la prensa a través de sus efectos: una propuesta metodológica. En D. Rodrigo-Cano, P.de-Casas-Moreno, \& P. Toboso-Alonso (Eds) Los medios de comunicación como difusores del cambio climático (pp. 107-122). Sevilla: Egregius.

Klein, N. (2015). Esto lo cambia todo. El capitalismo contra el clima. Barcelona: Paidós.

León, B., \& Bourk, M. (2018). Communicating sciencie and technology through online video. New York: Routledge.

Limón, D. \& Solís, C. (2014). Educación Ambiental y enfoque de género: claves para la integración, Rev. Investigación en la escuela, 33, 2014, 37-50.

Machuca, I. \& Rodrigo-Cano, D. (2018). La educomunicación ambiental hacia la transición energética desde la perspectiva de género. Estado del arte. En R. Fernández-Reyes \& D. Rodrigo-Cano (Eds). La comunicación de la mitigación y la adaptación al cambio climático (Pp. 49-58). Madrid: Ediciones Egregius.

Mancinas, R. (2012). El silencio mediático. Reflexión en torno a las razones de los medios de comunicación para no hablar del cambio climático. En R. Fernández-Reyes \& R. Mancinas (Ed). Medios de comunicación y cambio climático. Actas de las Jornadas Internacionales (pp. 233-248). Sevilla: Facultad de Comunicación de la Universidad de Sevilla.

Marta-Lazo, C. y Gabelas, J. A. (2016). Comunicación digital: Un modelo basado en el Factor R-elacional. UOC: Madrid.

Megías-Delgado, M., Baldallo-González, C., \& Maraver-López, P. (2018). Validez de la publicidad como recurso en la Educación Ambiental. Revista Mediterránea de Comunicación/ Mediterranean Journal of Communication, 9(2), 203-215. https://www.doi.org/10.14198/MEDCOM2018.9.2.11 
Meira, P. (2017). Retos para informar sobre el cambio climático a la sociedad. En Mónica Arto Blanco \& Pablo Meira (Eds) RESCLIMA: Aproximación ás claves sociais e educativas do cambio climático (pp.97-101). Ferrol: Aldine Editorial.

Meira, P., Barba, M., \& Castiñeiras, J.J. (2017). Crisis económica y profesionalización en el campo de la educación ambiental: comparativa 2007-2013 en Galicia. Educação e Pesquisa, 43(4), 1127-1146. https://dx.doi.org/10.1590/s1517-9702201703155092

Meira, P., González-Gaudiano, E., \& Gutiérrez-Pérez, J. (2018). Climate crisis and the demand for more empiric research in social sciences: emerging topics and challenges in environmental psychology / Crisis climática y demanda de más investigación empírica en Ciencias Sociales: tópicos emergentes y retos en Psicología Ambiental, Psyecology, 9(3), 259-271, DOI: 10.1080/21711976.2018.1493775

Meira-Cartea, P. A., Gutiérrez-Pérez, J., Arto-Blanco, M., \& Escoz-Roldán, A. (2018). Influence of academic education vs. common culture on the climate literacy of university students / Formación académica frente a cultura común en la alfabetización climática de estudiantes universitarios, Psyecology, 9(3), 301-340. DOI: 10.1080/21711976.2018.1483569

Ministerio de Agricultura, Alimentación y Medio Ambiente [España] (2014). Basado en materiales contenidos en el Quinto Informe de Evaluación del IPCC, Grupo de Trabajo II. Cambio Climático: Impactos, Adaptación y Vulnerabilidad. Fundación Biodiversidad, Oficina Española de Cambio Climático, Agencia Estatal de Meteorología, Centro Nacional de Educación Ambiental. Recuperado de https://goo.gl/btY3qi.

Murga-Menoyo, M. Á. (2018). La Formación de la Ciudadanía en el Marco de la Agenda 2030 y la Justicia Ambiental. Revista Internacional de Educación para la Justicia Social (RIEJS), 7(1), 37-52. https://doi.org/10.15366/riejs2018.7.1.002

Nagamini, E., \& Aguaded, I. (2018). La Educomunicación en el contexto de las nuevas dinámicas discursivas mediáticas [Editorial]. Revista Mediterránea de Comunicación/Mediterranean Journal of Communication, 9(2), 119-121. https://www.doi.org/10.14198/MEDCOM2018.9.2.27

Ojeda-Barceló, F., Gutiérrez-Pérez, J., \& Perales-Palacios, F.J. (2011). TIC y Sostenibilidad: Obstáculos y posibilidades para los educadores ambientales. Profesorado. Revista de Currículum y Formación de Profesorado, 15, 1, 263-313.

Ouariachi, T., Olvera, M.D., \& Gutiérrez, J. (2017). Analysis of online change games: exploring opportunities. Revista Electrónica de Investigación Educativa, 19(3), 104-114. https://doi. org/10.24320/redie.2017.19.3.1298

Peña-López, I. \& Padró-Solanet, A. (2017). Educación ambiental en un món de xarxes. En Diputació de Barcelona (Eds) Educació ambiental. D'on venim? Cap a on anem? (pp. 231-257). Barcelona: Col.lecció Estudis.

Picó Garcés, M.J. (2017a). Els mitjans de comunicació, aliats de l’educació ambiental. En Diputació de Barcelona (Eds) Educació ambiental. D'on venim? Cap a on anem? (pp. 219-230). Barcelona: Col.lecció Estudis.

Picó Garcés, M.J. (2017b). Periodismo ambiental. De la lucha ecologista al entorno digital. UOC. Barcelona.

Programa de las Naciones Unidas para el Desarrollo (2016). Objetivos de desarrollo sostenible. Recuperado de https://goo.gl/Rw4aiK

Reig, R., \& Labio, A. (2017). El laberinto mundial de la información. Barcelona: Anthropos/Siglo XXI.

Resclima (2018). A educación para o cambio climático no sistema educativo. Recuperado de: https://goo.gl/NHLD2B

Robert, Kates W., Parris, Thomas M., \& Leiserowitz, Anthony A. (2005). What is Sustainable Development? Goals, Indicators, Values, and Practice. Environment: Science and Policy for Sustainable Development, 47(3), 8-21. http://dx.doir.org/10.1080/00139157.2005.10524444

Rodrigo-Cano, D., \& Machuca-De-La-Rosa, I. (2018). La educomunicación ambiental, herramienta necesaria ante el cambio climático frente al greenwashing. En D. Rodrigo-Cano, P. de-Casas-Moreno, \& P. Toboso-Alonso (Eds), Los medios de comunicación como difusores del cambio climático (pp. 47-66). Sevilla: Egregius. 
Sarmiento, P. (2013). Bioética ambiental y eco pedagogía: una tarea pendiente. Acta bioethica, 19(1), 29-38.

Sauvé, L. \& Asselin, H. (2017). Educar para la ecociudadanía: contra la instrumentalización de la escuela como antesala del «mercado del trabajo». Teoría de la Educación. Revista Interuniversitaria, 29(1), 217-244. http://dx.doi.org/10.14201/teoredu291217244

Serantes, A. (2017). El cambio climático en los libros de texto. Cómo el sistema educativo contribuye a la construcción del saber sobre el cambio climático. En Mónica Arto Blanco \& Pablo Meira (Eds) RESCLIMA: Aproximación ás claves sociais e educativas do cambio climático (Pp.81-92). Ferrol, España: Aldine Editorial.

Severiche-Sierra, C., Gómez-Bustamante, E., \& Jaimes-Morales, J. (2016). La educación ambiental como base cultural y estrategia para el desarrollo sostenible. Telos, 18(2), 266-281.

Teso Alonso, G. (2016). Comunicación y representaciones del Cambio Climático: el discurso televisivo y el imaginario de los jóvenes españoles. Tesis Doctoral, Facultad de Ciencias de la Información, Universidad Complutense de Madrid, Madrid. Recuperado de https://goo.gl/JMZjYp)

Teso Alonso, G., Fernández- Reyes, R., Gaitán, J.A, Lozano-Ascencio, C., \& Piñuel, J.L. (2018). Comunicación para la sostenibilidad: el cambio climático en los medios. Madrid: Fundación Alternativas.

Toboso, P., De-Casas-Moreno, P., \& Rodrigo-Cano, D. (2018). \#EA26. Análisis de la iniciativa de educación ambiental. En D. Rodrigo-Cano, P. de-Casas-Moreno, \& P. Toboso-Alonso (Eds) Los medios de comunicación como difusores del cambio climático (pp. 145-162). Zaragoza: Egregius.

Vervoort, Joost M. \& Gupta, Aarti (2018). Anticipating climate futures in a $1.5^{\circ} \mathrm{C}$ era: The link between foresight and governance. Current Opinion in Environmental Sustainability, 31, 104-111.

Villalonga, C., \& Marta-Lazo, C. (2015). Modelo de integración educomunicativa de apps móviles para la enseñanza y el aprendizaje. Pixel-Bit. Revista de Medios y Educación, 46, 137-153. http:// dx.doi.org/10.12795/pixelbit.2015.i46.09

Zaragoza, M. F. (2018). El conocimiento del cambio climático a través de los mass media como herramienta de proyección. En D. Rodrigo-Cano, P. de-Casas-Moreno, \& P. Toboso-Alonso (Eds), Los medios de comunicación como difusores del cambio climático (pp. 29-46). Sevilla: Egregius. 